\title{
Operando Investigation of Energy Storage Material by FIB-SEM System
}

\author{
Xinwei Zhou ${ }^{1}$, Likun Zhu ${ }^{2}$ and Yuzi Liu ${ }^{1}$
}

${ }^{1}$ Argonne National Laboratory, Lemont, Illinois, United States, ${ }^{2}$ IUPUI, Indianapolis, Indiana, United States

It is well known that lithium-ion batteries are one of the most needed renewable energy storage technologies and being used in a variety of applications from smart phones to electric vehicles. However, the limited capacity and energy density of current battery materials cannot meet the requirement for long lasting applications, eg. long range driving. In order to impove the performence, various approaches were employed to reveal the working mechanism of the energy storage materials, especially during the charging and discharging process in real time. Different in situ characterization methods such as X-ray diffraction, X-ray absorption spectroscopy, coherent X-ray diffraction imaging and analytical transmission electron microscopy were used to monitor materials evolution during electrical cycling. Considering the dimensions of the energy storage materials fall to the range of the nanometers (single primary particles) to micrometers (secondary particles and some single crystal $\mathrm{Si}$, Ge and LiNiMnCo particles) and very often the materials change starting from surface, the FIB-SEM is the perfect platform to perform the energy storage materials study in the size range of a few number nanometers to a few micrometers. Also, SEM is one of the best tools to visualize the surface of samples in combination with different detectors. The FIB-SEM has been applied to study the single Li-ion battery cathode particles during electrochemical cycling ${ }^{1}$. The microstructural evolution of micrometer-sized single Sn and Ge particles during lithiation and delithiation along with electrochemical measurement was carried out on the improved experimental $\operatorname{setup}^{2,3}$.

The experiment was performed on a Zeiss FIB-SEM system. A particle was attached to a probe which is movable in 3 dimensions. The ionic liquid electrolyte was made by dissolving the Li salt, lithium bis (trifluoromethylsulfonyl) imide (LiTFSI) (Sigma-Aldrich), in a solvent of 1-butyl-1-methylpyrrolidinium bis (trifluoromethylsulfonyl) imide (P14TFSI) (Sigma-Aldrich). The electrolyte was loaded onto the Li metal plate in the sample chamber. The particle attached on the probe can be accurately moved to contact the electrolyte to form the single particle battery. Fig 1 shows voltage profile of single Ge particle and its morphology at different charging states. Fig. 1 a) shows the very smooth voltage profile of the single particle during cycling. The letters in Fig .1 a) indicate the charging states of Fig. 1 b), c) and d), pristine, lithiation, and delithiation respectively. The volume of the Ge particle increased a lot after the lithiation and shrink after the delithiation. More importantly, there is a big crack on the particle surface, which is the result of the dramatic volume change. In order to monitor the surface change of the particles, the ionic liquid electrolyte was replaced with solid electrolyte. From Fig. 2, the particle surface morphology change was clearly imaged. In addition to see the volume change of the Ge particles, the cracking of the particle from surface was observed as well. This example shows that the FIB-SEM is a perfect platform to study the energy storage materials at wide size range from nanometers to a few micrometers. It provides additional insight to understand the working mechanism of the energy storage materials.

This work was performed at the Center for Nanoscale Materials, a U.S. Department of Energy Office of Science User Facility, and supported by the U.S. Department of Energy, Office of Science, under Contract No. DE-AC02-06CH11357. 

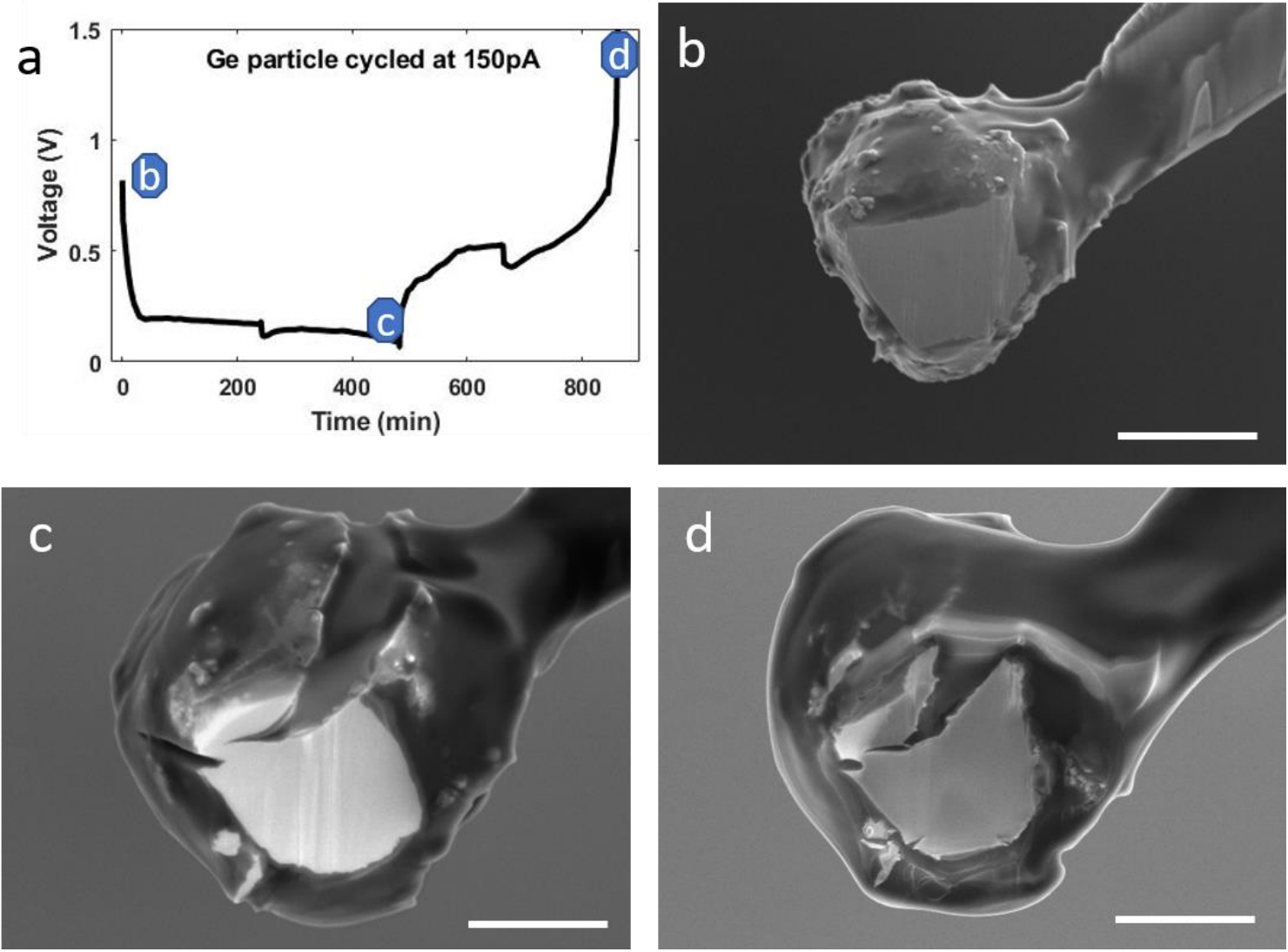

Figure 1. Germanium particle cycled at $150 \mathrm{pA}$ in ionic liquid electrolyte a. Voltage profile. b. Pristine particle c. Particle after discharging. d. Particle after charging. Scale bar: $1 \mathrm{um}$
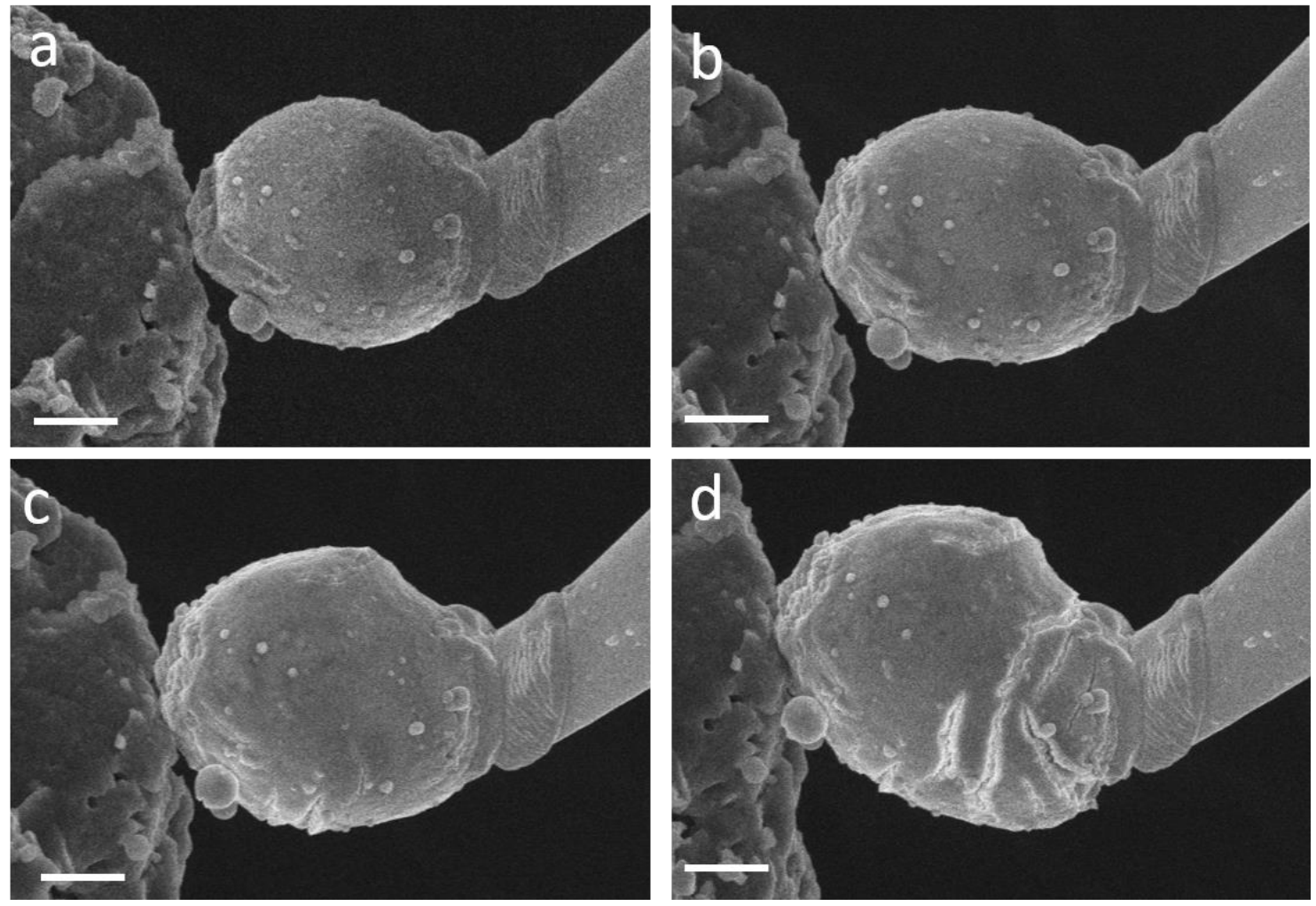
Figure 2. Lithiation of Ge particle with solid state electrolyte. a. Pristine particle; b - d Particle morphology at 20, 40 and 60 minutes of lithiation. Scale bar: 2 um

\section{References}

1. Miller Dean, C. Proff, J. G. Wen, P. Abraham Daniel and J. Bareño, Advanced Energy Materials 2013, 3, 1098-1103.

2. Zhou, X.; Li, T.; Cui, Y.; Fu, Y.; Liu, Y.; Zhu, L., In Situ Focused Ion Beam Scanning Electron Microscope Study of Microstructural Evolution of Single Tin Particle Anode for Li-Ion Batteries. ACS Applied Materials \& Interfaces 2019, 11 (2), 1733-1738.

3. Zhou, X.; Li, T.; Cui, Y.; Meyerson, M. L.; Mullins, C. B.; Liu, Y.; Zhu, L., In Situ Focused Ion BeamScanning Electron Microscope Study of Crack and Nanopore Formation in Germanium Particle During (De)lithiation. ACS Applied Energy Materials 2019, 2 (4), 2441-2446. 